diese allein bestimmend, z. T. sind auch wohl ältere Bruchränder durch die Erosion freigelegt und wieder herausgearbeitet worden (Nidda).

Es gehört nicht in den Rahmen dieser kurzen Skizze, auf die Veränderungen des Landschaftsbildes während der Diluvialzeit genauer einzugehen, und ich sehe um so lieber davon ab, als ich den Zeitpunkt für eine zusammenfassende Darstellung des Verlaufes dieser jüngsten Epoche in unserem Gebiete noch nicht für gekommen halte. Noch sind die Fragen nach der Gliederung, dem Alter, der Zusammengehörigkeit der einzelnen Terrassen keineswegs geklärt, und es wird hier noch vieler Spezialuntersuchungen bedürfen, ehe wir zu einer einigermaßen einheitlichen Auffassung kommen.

\title{
Die Eiszeit in den nordjapanischen Alpen.
}

Von K. Oseki (zurzeit in Berlin).

\author{
(Mit 5 Textfiguren.)
}

Über das Vorhandensein einer Eiszeit in Japan gehen die Meinungen noch sehr auseinander. Schon im Jahre 1903 hat der Professor der Geographie der Universität Tokyo, N. YAMASAKI, in den Formen der nordjapanischen Alpen glaziale Züge zu erkennen geglaubt und von Moränen, sowie von Gletscherschliffen gesprochen ${ }^{1}$ ). Aber M. Yokoyama, der Paläontologe zu. Tokyo, hat in marinen quartären Ablagerungen der Umgebung der Bai von Tokyo nicht Formen eines kühleren Klimas gefunden und bestreitet daher, daß in Japan eine Kälteperiode nachweisbar $\operatorname{sei}^{2}$ ). Der bekannte Mineraloge der Universität Tokyo, K. JIMBo, hat ferner in dem geologischen Kolloquium der Universität Tokyo wiederholt ausgesprochen, daß alle die von YAMASAKr in Betracht gezogenen Formen auch als Verwitterungserscheinungen gedeutet werden könnten. Die japanischen Alpinisten, z. B. TsuJimura ${ }^{3}$ ), hingegen haben sich der Ansicht von Professor Yamasaki angeschlossen und wiederholt von Karen, Moränen und anderen Eiszeitspuren berichtet. Die Ansicht von YокоYAMA ist dem deutschen Leserkreise durch LePSIUS ${ }^{4}$ ) mitgeteilt worden, während PENck auf Photographien der nordjapanischen Alpen, die ich ihm überbrachte, Kare und Endmoränenwälle erkannte ${ }^{5}$ ). Auch $\mathrm{H}$. SпмотомаI hat über solche auf Bildern erkennbare Gletscherspuren in

1) The Journal of the Geological Society of Tokyo. Vol. IX. 1903. (Japænisch.)

2) Climatic Changes in Japan since the Pliocene Epoch. Journal of the College of Science Tokyo. Vol. XXXII. Art. 5. 1911.

3) "Were the Cirques in Japan formed by Glacier? " The Journal of the Geological Society of Tokyo. Vol. XX. Nr. 238-239. 1913. (Japanisch.)

4) Keine diluviale Eiszeit in Japan. Geologische Rundschau III. 1912. S. 157.

5) Die Formen der Landoberfläche und Verschiebungen der Klimagürtel. Sitzungsberichte der Königlich preußischen Akademie der Wissenschaften. IV. 1913. 
den nordjapanischen Alpen berichtet $\left.{ }^{1}\right)$. Ich selbst hatte viermal Gelegenheit, die nordjapanischen Alpen zu besuchen, und habe an einzelnen Gipfeln deutlich Eiszeitspuren beobachtet, worüber ich, einer Anregung von Professor PENCK folgend, hier berichten möchte.

Die nordjapanischen Alpen oder das Hidagebirge bilden das östliche Randgebirge des sich in $1000 \mathrm{~m}$ Meereshöhe ausdehnenden Hidaplateaus, des höchsten Plateaus der japanischen Inseln. Das Schichtstreichen ist im Plateau und Gebirge das gleiche; im Süden von SW. nach No., weiter gegen N. mehr östlich; es läuft also im ganzen sehräg zu der nordsüdlichen Längserstreckung des Gebirges.

In geologischer, sowie topographischer Hinsicht lassen sich im Hidagebirge zwei Teile unterscheiden; ein südlicher von vorwiegend vulkanischem Aufbau, und ein nördlicher, meistenteils aus paläozoischen Gesteinen zusammengesetzter. Die Grenze beider wird orographisch am besten durch den nur $1583 \mathrm{~m}$ hohen $\mathrm{Abō} \mathrm{Pa} B$ (Fig. 5-15) gezogen, welcher einen sehr tiefen Einschnitt im Gebirge bildet. Allerdings läßt er den $2458 \mathrm{~m}$ hohen tätigen Vulkan Iwō Dake (Fig. 5-14) bei dem nördlichen Teil, während sonst die Vulkanberge auf den südlichen Teil der nordjapanischen Alpen beschränkt sind. Wollte man alle Vulkanberge in dieser Gruppe vereinigen, so müßte man die Grenze beider Gebirgsteile nördlich des Iwō Dake über den $2094 \mathrm{~m}$ hohen Nakao Paß (Fig. 5-13) ziehen, welcher nur eine geringe Einsenkung des Kammes darstellt. Der durch die abtragenden Kräfte wesentlich erniedrigten alten paläozoischen Oberfläche des Südteiles ist eine nordsüdlich verlaufende Reihe von Vulkankegeln, die Norikura Gruppe (Fig. 5-16), aufgesetzt. Im Norden finden wir dagegen die alte Oberfläche in weit größerer Höhe; jungvulkanisches Gestein ist hier nur wenig vorhanden. Die Bezeichnung "nordjapanische Alpen « paßt eigentlich nur auf den nördlichen Teil des Hidagebirges; denn nur hier zeigen Oberflächengestaltung, Klima und Pflanzen alpinen Charakter.

In diesem Teil des Gebirges fällt uns vor allem der sehr steile Abfall nach Osten auf. Hier zieht der große Graben; nach Naumanns Benennung "fossa magna «, von NNW. nach SSO. entlang, der die beiden tektonischen Bogen Japans trennt. An diesen Graben knüpft sich eine Reihe von Vulkanen, die Fujikette, während an seinen Rändern sich Horstund Halbhorstgebirge erheben.

Der Ostabhang des Hidagebirges wird entwässert durch drei Flüsse, den Hime Kawa, Takasegawa und Azusagawa. Die beiden letzteren kommen in tiefeingeschnittenen Tälern aus dem Inneren des Gebirges heraus und fließen in den Sai Kawa, der weiterhin in seinem Unterlauf Shinanogawa heißt. Auf der anderen Seite des Gebirges entspringen Jogwanjigawa und. Jinzugawa, die ebenfalls tief eingeschnitten sind. Parallel zum Ostabfalle gelangt der Kurobegawa zum Toyama Golf.

1) Die diluviale Eiszeit in Japan. Zeitschrift der Gesellschaft für Erdkunde zu Berlin. 1914. Nr. 1. 
Von dem südlichen Teil des Hidagebirges fließen zahlreiche kleine Bäche zum Jinzugawa und Azusagawa, während nach Süden der Kisogawa mit dem Nebenfluß Mashitagawa oder Hidagawa in den Ise Golf an der Südküste von Honshu fließen.

Über das Vorkommen der einzelnen Gesteinsarten sei folgendes bemerkt: Wir finden im nördlichen Gebiet archäisches Gestein, vor allem Gneis, im mittleren und südlichen Teile des Hidagebirges dagegen paläozoische Schichten (meistenteils Tonschiefer, Hornstein) und ältere Eruptivgesteine wie Granit, Porphyr und Porphyrit. Ganz im Süden treten dann jungvulkanische Gesteine auf und greifen auch auf den nördlichen Teil des Hidagebirges über.

Kein Gipfel Japans ragt gegenwärtig über die Schneegrenze hinaus; selbst der $3778 \mathrm{~m}$ hohe Fujisan hat keine perennierenden Schneefelder. Nur an geschüitzten Stellen der nordjapanischen Alpen bleibt der Schnee in kleinen Flecken das ganze Jahr über liegen. Es fallen im Hidagebirge jährlich etwa $2500 \mathrm{~mm}$ Niederschlag, und zwar zum größten Teil als Schnee. Die nordwestliche Seite ist stärker benetzt als die südöstliche, die wahrscheinlich weniger als $2000 \mathrm{~mm}$ Niederschlag empfängt. Jedoch sind wir über die meteorologischen Verhältnisse schlecht unterrichtet, da im Gebirge meteorologische Stationen gänzlich fehlen. Beobachtungen sind lediglich in einiger Entfernung vom Fuße, nämlich in den Städten Matsumoto im Osten und Takayama im Westen angestellt worden.

Das Hidagebirge zerfällt, wie wir gesehen, in einen nördlichen und in einen südlichen Teil. Der erstere wird durch das Längstal des Kurobegawa und den Längstalzug des Takasegawa und Azusagawa in drei parallele Ketten zerlegt, die wir westlichen Zug oder Tateyamakette (I), Zentralzug (II) und östlichen Zug oder Otenjokette (III) nennen wollen. Der Zentralzug bildet keine geradlinig fortlaufende Kette, sondern besteht aus zwei Kammpartien, zwischen die sich der $2541 \mathrm{~m}$ hohe Harinokipaß (Harinoki Toge) wie ein Längspaß einschaltet. Den nördlichen Kamm möchte ich die Kette des Shirouma Dake und den südlichen Zug die Kette des Yarigatake nennen. Wir stellen die Gliederung des Gebirges in folgender Tabelle dar:

A. Nördliches Hidagebirge (Hauptkette)

a) Westlicher Zug oder Tateyamakette.

b) Zentralzug

I. Die Shirouma Dake Kette

II. Die Yarigatakekette

c) Östlicher Zug oder Otenjōkette

B. Südliches Hidagebirge, d. i. Hauptteil der Norikura Vulkangruppe.

Wir betrachten nunmehr die eiszeitlichen Gletscherspuren in den einzelnen Teilen der nordjapanischen Alpen.

a) Westlicher Zug oder Tateyamakette.

Die Tateyamakette, in der ein berühmter Wallfahrtsort gelegen ist, und die im Sommer von vielen Touristen besucht wird, erhebt sich mit mehr 
als zehn Gipfeln über $2600 \mathrm{~m}$; der Hauptgipfel, jedoch nicht der höchste, ist der Berg Oyama (Fig. 5-3) $2992 \mathrm{~m}$, den Professor YaMaSaKI und ich im Jahre 1904 bestiegen haben. Wir fanden hier am Westabhang ein Kar, dem in etwa $2500 \mathrm{~m}$ Höhe zwei Reihen Endmoränen vorgelagert sind. Fig. 1 gibt die Szenerie nach einer Zeichnung von mir wieder. Kürzlich hat Tsusrmura auch in "The Journal of the Geological Society of Tokyo « (Vol. XX, Nr. 239) eine lehrreiche Abbildung der Ostseite der Tateyamakette bis zum $2998 \mathrm{~m}$ hohen Tsurugigatake gegeben, welche

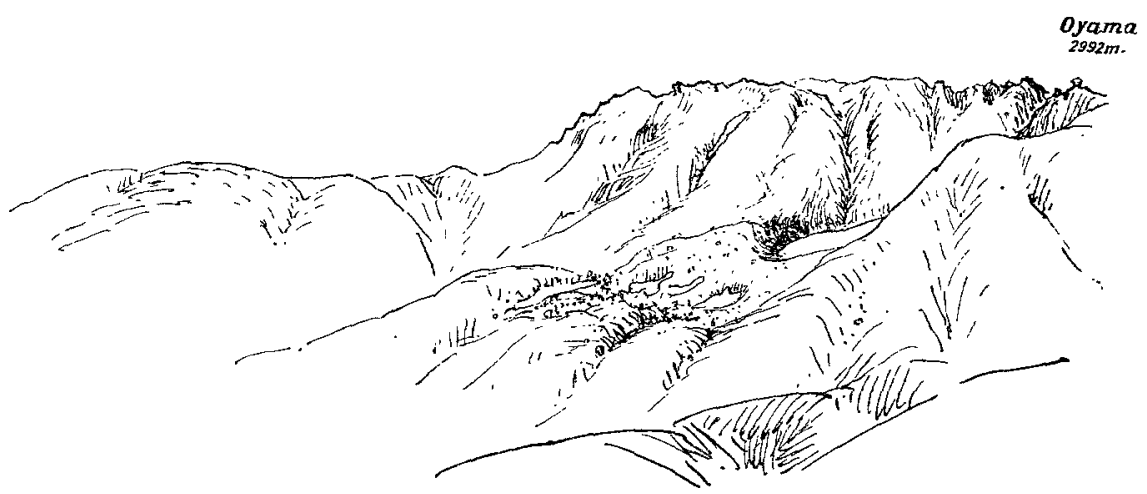

Fig. 1. Kar am Westabhang des Oyama mit 2 vorgelagerten Endmoränezügen.

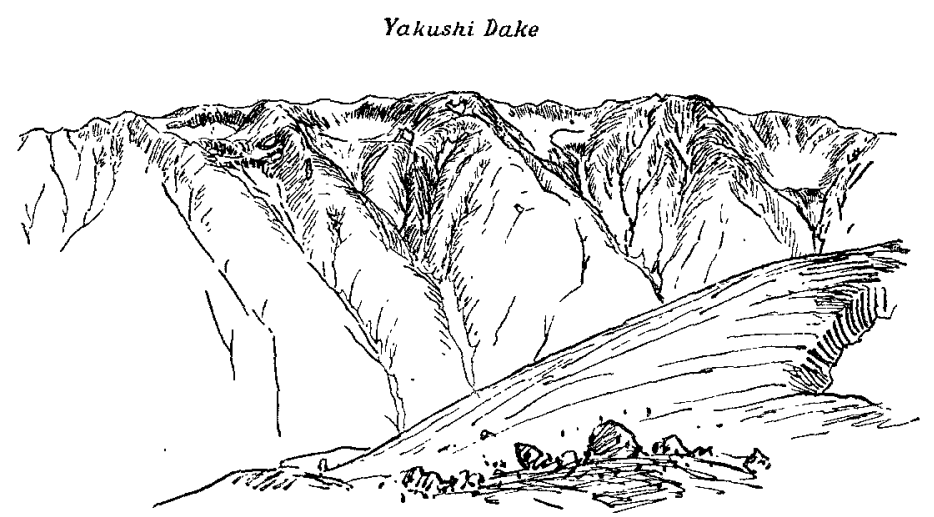

Fig. 2. Vier Kare an der Ostseite des Yakushi Dake.

deutlich erkennen läßt, daß auch dieser Abhang in seiner ganzen Länge mit Karen besetzt ist. Auf Bildern, welche im letzten Sommer von meinem Freunde K. Tomono aufgenommen worden sind, kann ich ganz sicher an einem Berge, Yakushi Dake, Kare erkennen. Ich habe danach die folgende Skizze gezeichnet. Fig. 2 zeigt die Ostseite des Kammes von Yakushi Dake (2986 m) gegen das Tal des Kurobegawa. Man sieht hier vier Kare nebeneinander, deren Boden etwa $2800 \mathrm{~m}$ hoch liegt. 


\section{b) Zentralzug.}

I. In der nördlichen Kette des Zentralzuges, der Shirouma Dake Kette, erhebt sich auch eine Zahl von Gipfeln über $2600 \mathrm{~m}$. Die Frage nach der Vergletscherung wurde im Jahre 1903 durch Professor Yamasaris Besuch des Shirouma Dake oder Hakuba Dake (d. i. Weißpferd Gipfel) (Fig. 5-1) aufgerollt. Er fand dort Moränen und Gletscherschliffe. Ein Jahr später fanden Professor YAMASAKI und ich auf einer gemeinschaftlichen Reise am Ostabhang (2520 m) des Harinoki Dake (Fig. 5-4) ein großes Kar, bis zu dessen Schwelle wir emporstiegen, als wir den Harinokipa $B$ überschrittén. Die Bodenfläche dieses Kares ist schüsse]förmig und wird gegen Osten von den sehr steilen Felswänden des Harinoki Dake überragt. Gletscherschliffe haben wir bei unserem flüchtigen Besuch nicht gefunden.

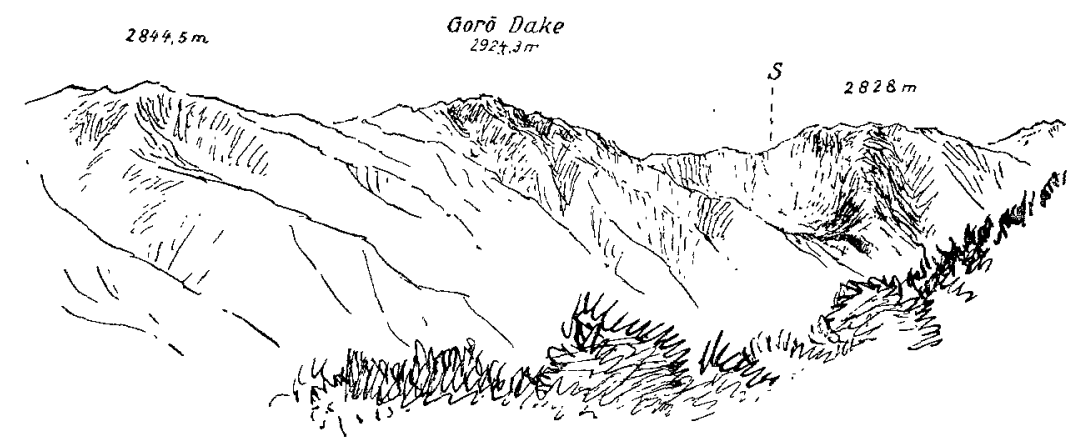

Fig. 3. Kar an der Westseite des Gorō Dake.

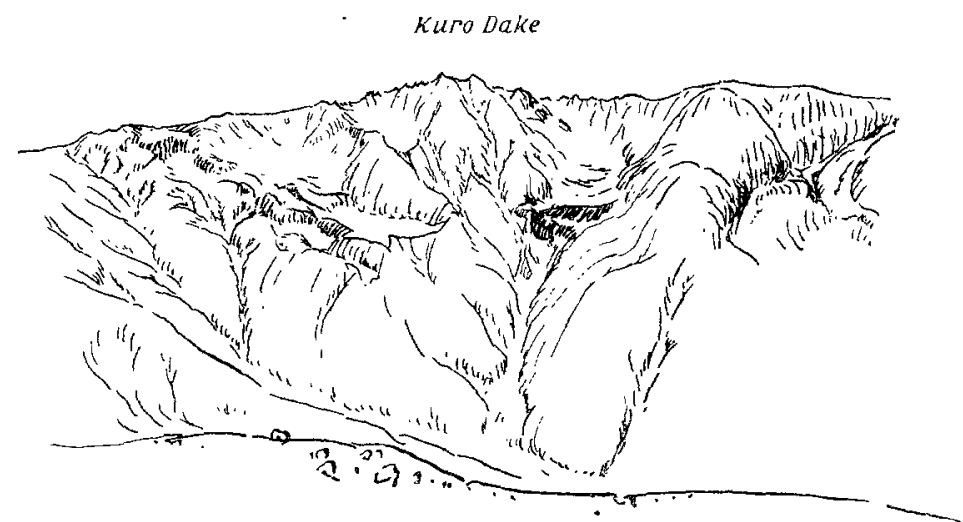

Fig. 4. Drei Kare am Ostabhang der Kuro Dake.

II. Die Gipfelhöhen der Yarigatakekette sind durchschnittlich bedeutender als die der anderen Gruppen und überschreiten in der Regel $2900 \mathrm{~m}$. Am Goro Dake (2924 m oder Fig. 5-8) sahen Professor YAMASAKI und ich im Jahre 1904 ein großes Kar mit Westexposition, 
dessen Bodenhöhe nach der provisorischen Generalstabskarte $1: 50000$ etwa $2540 \mathrm{~m}$ ist. Fig. 3 gibt es nach einer Zeichnung von mir wieder. Leider war es uns nicht möglich, in das Kar selbst zu gehen. Seither haben Alpinisten auch an anderen Bergen Kare und Moränen gefunden. Fig. 4, deren Original auch ich Herrn TomoNo verdanke, zeigt den Ostabhang des Kuro Dake (2978 m (Fig. 5-7)), eines Gipfels auf einer Kette, die sich unweit des Gorō Dake vom Hauptkamm loslöst und parallel zu ihm nach Norden läuft, von ihm getrennt durch das Tal des Higashizawa, eines Seitentales vom Kurobegawa. Man hat in der Mitte ein großes Kar mit abgestuftem Boden, dessen Höhe ich nach der Karte auf $2800 \mathrm{~m}$ veranschlage, links liegt ein kleineres Kar, rechts ein größeres, dessen steile Südseite wandähnlich verläuft. Das Bild ist vom schon gezeichneten großen Kare des Gorō Dake an der anderen Seite des Higashizawa aufgenommen.

Von Gletscherspuren am weiter südlich gelegenen Yarigatake (3178m) hat bereits Professor Pewck gesprochen. Eine Ansicht des Yarizawatales am Oberlaufe des Azusagawa unter dem südöstlichen Abhang von Yarigatake, d. i. Spießgipfel (Fig. 5--12), zeigt einen blocküberstreuten Wall (in etwa $2700 \mathrm{~m}$ Höhe), den Professor Penck nur als Endmoräne deuten kann. Weiter südlich treffen in der Gruppe des wild zerrissenen Hodaka . Dake (Fig. 5-11) (der höchste Gipfel OkuHodaka Dake $3103 \mathrm{~m}$ ) ausgedehnte Schneefelder. Auch hier dürften Spuren alter Gletscher zu gewärtigen sein.

Aus dem östlichen Zug des Hidagebirges, der im $2922 \mathrm{~m}$ hohen Otenjo Dake, d. i. Großen Tenjo Dake (Fig. 5-9) gipfelt, sind eiszeitliche Gletscherspuren bisher noch nicht gefunden worden. Die japanische Generalstabskarte verzeichnet am. Higashi-Tenjo Dake (Fig. 5-10), d. i. östlichen Tenjō Dake (281 m), deutliche Karformen.

Auch aus dem südlichen Hidagebirge, wo sich die Vulkane Norikura Dake (3026 m) (Fig. 5-16) und Ontake (3185 m) (Fig. 5-17) erheben, sind Gletscherspuren noch nicht bekannt geworden.

Ich fasse das Gesagte wie folgt zusammen:

1. Gletscherspuren sind in Japan bisher nur in Gestalt von Karen und Moränenwällen im nördlichen Hidagebirge gefunden. Dem Akaishigebirge (südjapanische Alpen), deren Hauptgipfel Akaishi San $3092 \mathrm{~m}$ hoch ist, glauben Alpinisten auch Gletscherspuren gefunden zu haben, doch vermag ich dies nicht aus eigener Anschauung zu bestätigen.

2. Die Gletseherspuren im nördlichen Hidagebirge sind nur unbedeutend. In der Eiszeit haben sich lediglich kleine Hängegletscher bis zu einer Höhe von etwa $2500 \mathrm{~m}$ über dem Meeresniveau herabgezogen. 


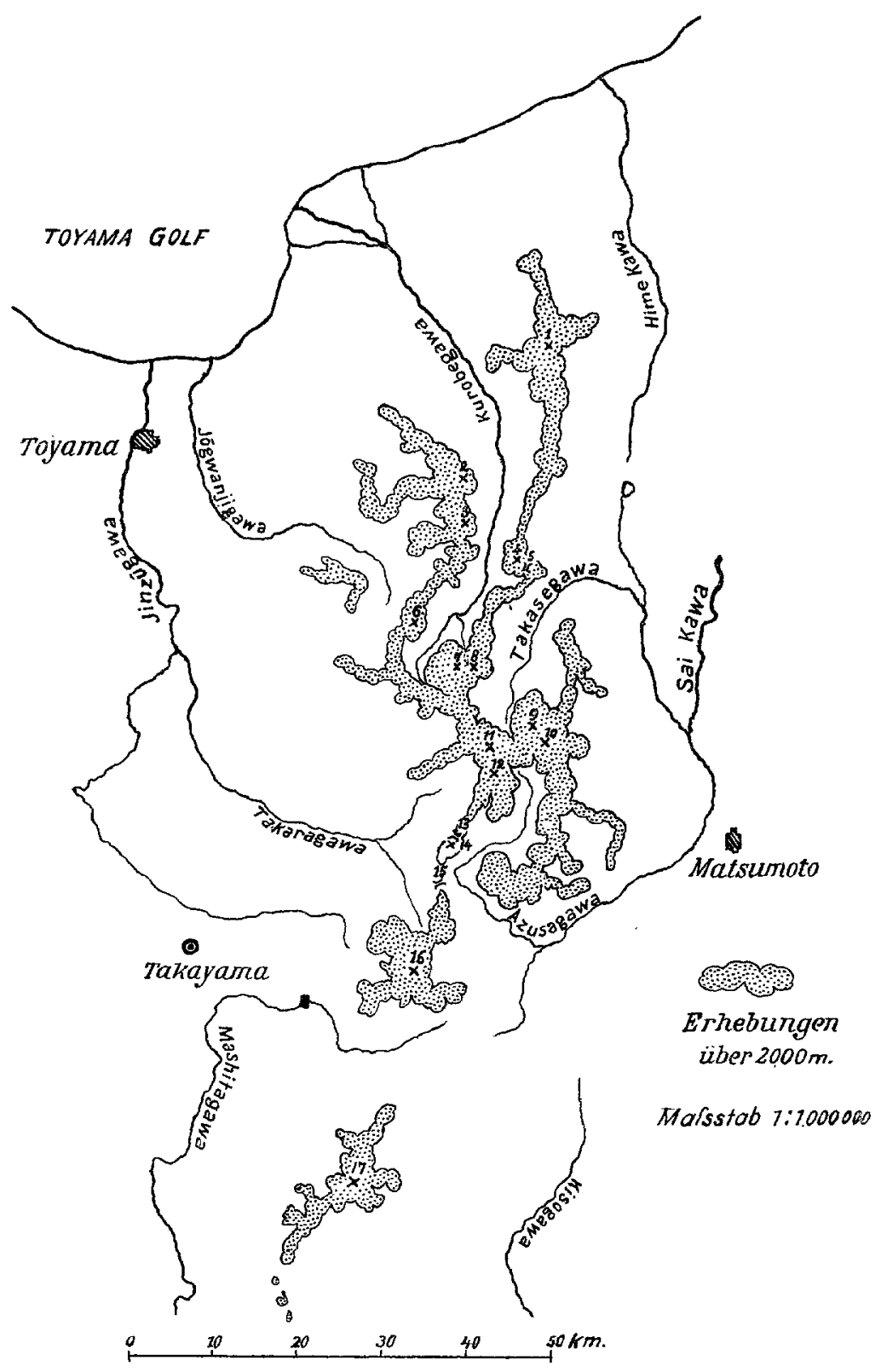

Fig. 5.

Übersichtskarte der nordjapanischen Alpen.

(Erklärung der Figur auf Seite 353.) 
3. Moränen mit gekritzten Geschieben, Gletscherschliffe und Rundhöcker sind bisher noch nicht mit Sicherheit in den Karen nachgewiesen worden. Die Höhe des Karbodens liegt an der niedrigsten Stelle etwa $2500 \mathrm{~m}$ über dem Meeresniveau, und in dieser Höhe möchte ich die Lage der eiszeitlichen Schneegrenze annehmen. Das stimmt mit dem Ergebnis von H. Simotomai. Da nun der höchste Gipfel des Gebirges, Yarigatake $3178 \mathrm{~m}$, die heutige Schneegrenze nicht erreicht, so müssen wir auf eine Depression der glazialen Schneegrenze von mehr als $700 \mathrm{~m}$ schließen $\left.^{1}\right)$.

\title{
Erklärung zu Fig. 5.
}
1. Shirouma Dake $2933 \mathrm{~m}$
10. Higashi Tenjō Dake $2811 \mathrm{~m}$
2. Tsurugigatake $2998 \mathrm{~m}$
12. Yarigatake $3178 \mathrm{~m}$
3. Oyama $2992 \mathrm{~m}$
11. Hodaka Dake $3103 \mathrm{~m}$
4. Harinoki Dake $2820 \mathrm{~m}$
13. Nakao Paß $2094 \mathrm{~m}$
5. Harinoki $\mathrm{Pa} 2541 \mathrm{~m}$
14. Iwō Dake $24.58 \mathrm{~m}$
6. Yakushi Dake $2986 \mathrm{~m}$
15. Abō $\mathrm{Pa} 1583 \mathrm{~m}$
7. Kuro Dake $2978 \mathrm{~m}$
16. Norikura Dake $3026 \mathrm{~m}$
8. Gorō Dake $2924 \mathrm{~m}$
17. Ontake $3185 \mathrm{~m}$.

\section{Beiträge zur Kenntnis von Tektonik und Glazial der bolivischen Ostcordillere.}

\author{
Von Dr. Th. Herzog (Zürich).
}

(Mit 10 Textfiguren.)

In dieser Arbeit lege ich die geologisch-tektonischen Beobachtungen nieder, welche ich gelegentlich einer von Oktober 1910 bis November 1911 in Bolivien durchgeführten botanisch-geographischen Forschungsreise gesammelt habe. Dieselben können, wenn auch von einem Nichtfachmann aufgezeichnet, immerhin dazu dienen, die Kenntnis der noch sehr unvollkommen erforschten östlichen Cordillerenzüge Bolivias zu erweitern und zu mancher neuen Fragestellung anregen. Leider ergibt

1) Weit außerhalb unseres Gebietes, nämlich nahe am Ostfuße des Hidagebirges, in nur etwa $800 \mathrm{~m}$ Meereshöhe, hat, wie ich aus Japan höre, Professor HeTtNeR in einer Schuttablagerung am Ufer des Azusagawa einen großen gekritaten Block von Biotitgranit gefunden. Auf der Photographie dieses »Hettner-Steins «, welche der Professor der Geographie T. Katō mir geschenkt hat, sieht man in der Tat ganz deutlich Schrammen. Sollte es sich um solche glazialen Ursprungs handeln, so würden wir im Tale des Azusagawa einen etwa $40 \mathrm{~km}$ langen Talgletscher anzunehmen haben, also einen viel größeren Eisstrom, als wir bisher feststellen konnten, oder müßten an eine Verschwemmung glazialer Blöcke glauben, wie sie nur ausnahmsweise vorkommt.

Geologische Rundschau. V. 